\title{
Spaceborne GPS \\ Current Status and Future Visions
}

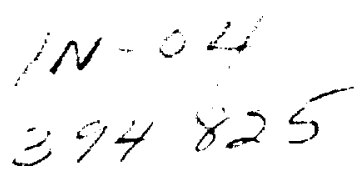

\author{
Frank H. Bauer, Kate Hartman and E. Glenn Lightsey \\ NASA Goddard Space Flight Center \\ Greenbelt, Maryland, 20771 \\ 301-286-8496 \\ frank.bauer@gsfc.nasa.gov
}

\begin{abstract}
The Global Positioning System (GPS), developed by the Department of Defense, is quickly revolutionizing the architecture of future spacecraft and spacecraft systems. Significant savings in spacecraft life cycle cost, in power, and in mass can be realized by exploiting Global Positioning System (GPS) technology in spaceborme vehicles. These savings are realized because GPS is a systems sensor-it combines the ability to sense space vehicle trajectory, attitude, time, and relative ranging between vehicles into one package. As a result, a reduced spacecraft sensor complement can be employed on spacecraft and significant reductions in space vehicle operations cost can be realized through enhanced onboard autonomy. This paper provides an overview of the current status of spaceborne GPS, a description of spaceborne GPS receivers available now and in the near future, a description of the 1997-1999 GPS flight experiments and the spaceborne GPS team's vision for the future.
\end{abstract}

\section{INTRODUCTION}

GPS technology holds great promise for terrestrial as well as space-based users. The world is just beginning to understand the tremendous benefits and great potential that this technology can deliver to the military and civilian transportation industry. Safer air travel, improvements in search and rescue systems, improved Earthquake monitoring, tractor-trailer tracking and enhanced farming techniques are just some of the terrestrial-based spinoffs from this technology. Handheld GPS receivers are now available for less than $\$ 100$ and millions are being sold each year. The benefits of this technology on Earth is extensive. The benefits are equally extensive for spacecraft and space systems. Significant reductions in spacecraft costs, improvements in spacecraft autonomy and new, exciting scientific opportunities can be accomplished through the infusion of this technology on spacecraft and spacecraft constellations of the future.
While this technology holds great promise, its incorporation on spacecraft has been delayed for several reasons. See figure 1. The tremendous success of the very lucrative terrestrial GPS market has, in fact, stifled the development of spaceborne GPS receivers. Companies with GPS expertise are more interested in the lucrative terrestrial-based GPS market. They are not interested in diverting their GPS talent on the relatively small space-based market. This has restricted the development of spaceborne receivers to meet the demands of future spacecraft requirements.

In addition to the above problem, there are many technical challenges that must be overcome before space-borne GPS bears all its fruit. GPS receivers that are used in space are very different than their terrestrialbased cousins. The high speeds of low Earth orbiting spacecraft result in signal doppler and doppler rate which are significantly higher than what is observed on the ground. Also, the GPS satellites rise and set on low Earth orbiting vehicles much faster than terrestrial based users (approximately 45-50 minutes versus 6 hours). These differences result in a significantly larger search space for spaceborne GPS requiring a much faster solution. Figure 1 outlines these problems and describes the technological "stairsteps" that the spaceborne GPS community needs to climb before spacebome GPS has fully matured.

The spaceborne GPS community must expediently overcome the GPS technology hurdles shown in figure 1 and develop a stable of robust spaceborne GPS receivers that meet future mission requirements. To this end, the GPS team at the Goddard Space Flight Center (GSFC) has been fostering government/university/industry partnerships in spaceborne GPS technology. The objectives of these partnerships are to promote the development and use of spaceborne GPS through a fourpronged program. The elements of this program include:

1) The development of spaceborne GPS receivers which satisfy the future spacecraft mission requirements. 
2) The development of the techniques required to integrate this technology on spacecraft in a cost effective manner.

3) The validation of this technology through a series of flight experiments; and,

4) The development of enhanced autonomy techniques such as autonomous orbit control and formation flying.

The GPS technology program being accomplished at NASA Goddard encompasses the use of GPS primarily as an engineering sensor as compared to using GPS for science measurements. As an engineering sensor, GPS determines spacecraft attitude, relative and absolute orbit position, and time. When used as a science instrument, GPS performs gravity, atmospheric sounding, ocean reflection, and ionospheric sounding measurements. While the science and engineering aspects of using GPS in space follow somewhat similar hardware development paths, the receiver robustness, data requirements, and receiver operation in an engineering application is very different from its use as a science instrument. This is primarily because the engineering function must maintain space vehicle health and is geared towards reducing space mission costs through reduced sensor complements and enhanced vehicle autonomy. To achieve these objectives, the GPS receiver must provide real-time, autonomous, onboard support to the spacecraft. This contrasts with the objectives of GPS science applications which demand extensive post processing of the data to glean as much information from the science data as possible. This data reduction is currently performed by a cadre of ground operations personnel. Moreover, the GPS science instrument is not required to maintain mission critical functions; thus, the electronics hardware embedded in the receiver and the

\section{Roadblocks Impeding Space-Based GPS Technology}

\footnotetext{
- Perception that GPS technology is mature and requires no research and development effort to fly in spece

- More lucrative GPS receiver marteet for terreatrial applications

- Differences between space-based \& ground-based GPS reception

- Require numerous flight experiments to "climb the technological stairsteps"

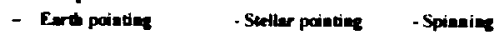

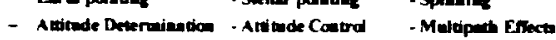

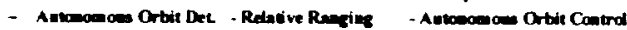

- For menios tyiar CEOrEO Ope - Precise Timieg

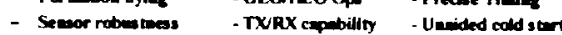

Figure 1: Roadblocks to Space-Based GPS algorithms used to obtain the GPS data can be very different from a GPS engineering device.

\section{VISION FOR SPACEBORNE GPS}

The current vision for spaceborne GPS technology is encapsulated in figure 2.

\section{Space-Based GPS Technology: \\ Vision of Highly Autonomous Spacecraft}

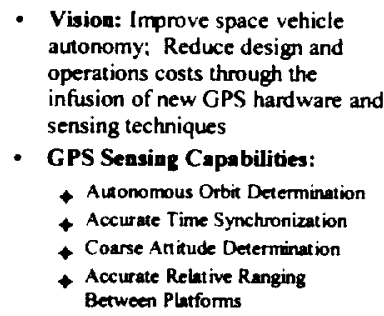

- Vision: Improve space vehicle autonomy: Reduce design and operations costs through the infusion of new GPS hardware and sensing techniques

- GPS Sensing Capabilities:

- Autonomous Orbit Determination

- Aocurate Time Synchronization

+ Coarse Atritude Determination

- Accurate Relative Ranging

Between Platforms

\section{Figure 2: Spaceborne GPS Vision}

As shown above, the vision embodies the exploitation of GPS as a systems sensor-capable of determining spacecraft trajectories autonomously, delivering precise time synchronization to spacecraft electronics, sensing vehicle attitude and measuring the relative distance between space vehicles. Achieving the vision outlined in figure 2 will take many years to complete. The current generation of receivers, being installed on today's flight experiments, will definitely open new doors in space vehicle design and autonomy. However, they will not fully achieve the revolutionary changes in spacecraft sensing and autonomy outlined in the vision statement depicted in figure 2 . The GPS technology roadmap, shown in figure 3 , illustrates that this will require approximately 10 years of concentrated development effort and probably three generations of flight receiver designs - the first generation of which is available today.

The current generation (first generation) of engineering receivers have been installed on several spacecraft and are being put through a battery of tests to validate their on-orbit performance and to understand their limitations. The information gleaned from these tests will be used to give GPS users the information they need to successfully integrate these current generation receivers onto their spacecraft and will also give the GPS development team the knowledge required to improve future receiver designs.

The second generation of receivers, expected to be available at the beginning of the next millennium, will be miniaturized versions of their predecessors, fabricated 


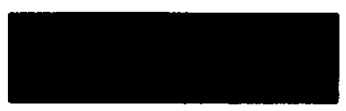

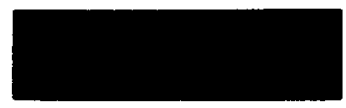

- Radiation Hardened

-Algorithms adapted for space

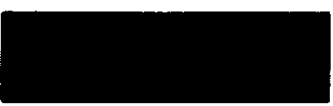

-Robust algorithms

- Modular S/W

- Miniaturized H/W

(GPS on Board)

-Enhanced initialization

- Navigation (500 m Real Time, $60 \mathrm{~m}$ Post Process)

-Precise Time through Bus ( $2 \mathrm{~ms}$ ) -Attitude Determination (0.25-0.1 $\left.1^{\circ}\right)$
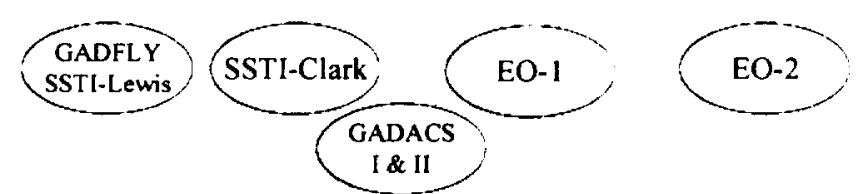

- Navigation (5m WAAS)

- Relative Navigation ( $10 \mathrm{~m}$ )

- Ops above the constellation

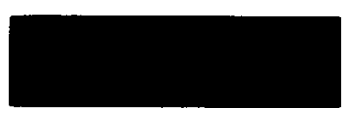

-TX/RX Capability -GPS on a Chip - Cold start w/o Ephemeris

-Dual Freq. Miniaturized

- GEO \& HEO Applications - Relative Navigation $(5 \mathrm{~cm})$

-Formation Flying

-Microsat Constellations

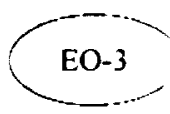

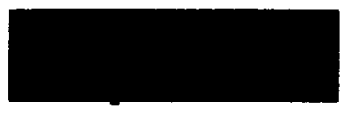
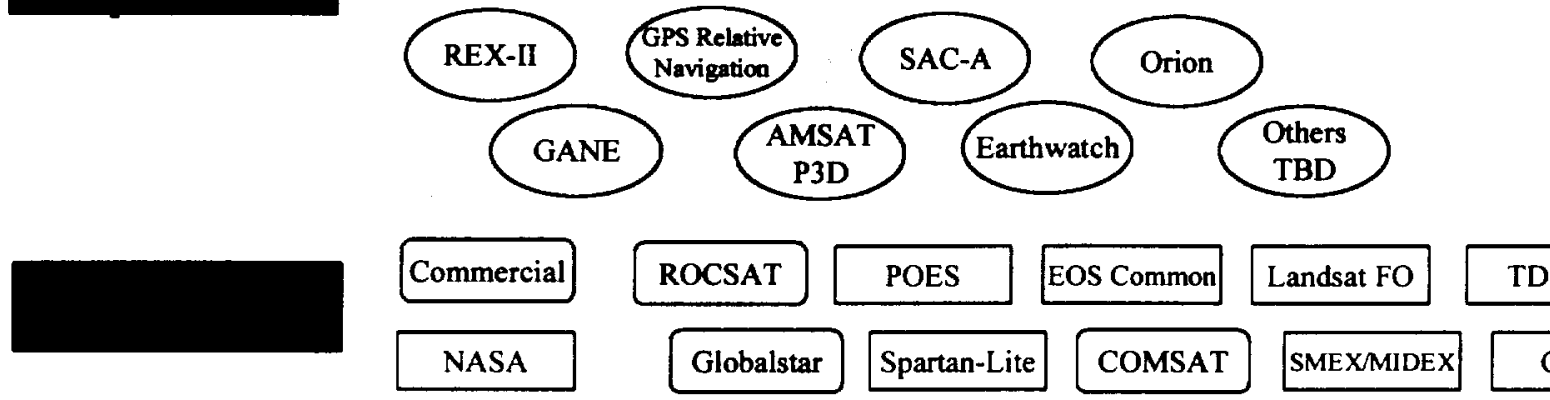

Landsat FO

TDRSS

Key

Figure 3: GPS Receiver Technology Roadmap

on multi-chip modules. They will include modular object oriented software and robust algorithms to significantly improve their operation in space. The receiver will be capable of quick ( 5 minute) cold start initialization without ground intervention. This new receiver set will enable real-time space vehicle navigation to meter performance levels using the Wide Area Augmentation System (WAAS) and will provide relative navigation knowledge to 10 meters.

The third generation of spaceborne receivers will enable inexpensive micro-spacecraft. Electronics technology improvements will allow the third generation receiver to be further miniaturized. A transmit/receive capability will be included in this generation receiver to enable autonomous formation flying. The "GPS-on-a-Chip" vision, proposed in 1995 by Goddard, Stanford University, and JPL, should be fully realized with this receiver. Robust use of GPS in Geostationary Earth Orbit (GEO) and High Earth Orbit (HEO) will be realized with this generation of receivers.

\section{GPS-AN ENABLING TECHNOLOGY}

Incorporation of GPS on space vehicles enables three primary technologies: space vehicle autonomy, ground station automation and virtual spacecraft constellations. Without GPS, all three of these technologies could grow and mature over time-but not without a high ground operations cost penalty and long-term development schedules. With GPS, each of these technologies become spacecraft breakthrough technologies, providing low cost, attractive opportunities for missions of the future.

Space Vehicle Autonomy-Low cost autonomous navigation, on-board maneuver planning and autonomous constellation control all become feasible when GPS is employed. Traditionally, spacecraft navigation would be accomplished on the ground through ranging and trajectory determination techniques. Planning and controlling the orbit of a single spacecraft from the ground is labor intensive. Performing these functions from the ground on several spacecraft simultaneously is extremely complex and introduces an overwhelming ground personnel requirement. This would place a considerable burden on the ground 
operations team who must not only ensure that all spacecraft maintain proper orbital spacing, but also monitor spacecraft states of health while collecting all pertinent data required to achieve overall mission success. The time, orbit and attitude data, obtained from GPS, enables spacecraft system developers to accomplish autonomous orbit maneuver planning and autonomous stationkeeping maneuvers on-board the spacecraft. This results in a substantial reduction in mission operations costs.

Other spacecraft autonomy technologies enabled using GPS are low cost, standardized spacecraft timing systems through the spacecraft data bus, vehicle attitude determination and attitude control, and autonomous data transmissions over ground stations. Miniaturized copies of a GPS receiver can also serve as the heart of an autonomous micro-sciencecraft providing attitude and orbit sensing, attitude commanding, orbit control, command and data handling services, and science instrument timing all in one package.

Virtual Platforms- Developing the technology to produce virtual platforms will be a long range challenge. Similar to its enabler-GPS technology-there are several technological hurdles that must be overcome to go from autonomous navigation and constellation control to one and two way formation flying and finally to virtual platforms (see ligure 4). Despite these hurdles, the Earth and space science community has already started defining the new science measurements and science missions that can be performed using clusters of spacecraft flying in formation in virtual platforms.

One technological hurdle critical to enable autonomous formation flying is the (transmit/receive) space vehicle crosslink system that is to be incorporated in the third generation GPS receiver (see figure 3 ). When the third generation receiver is coupled with autonomous onboard maneuver planning and orbit control software, autonomous relative ranging between space vehicles and formation flying becomes feasible. Using the crosslinkGPS receiver/transmitter as an enabling step, relative ranging and formation flying technology is expected to revolutionize both manned and unmanned space vehicle operations in the near future.

Once formation flying techniques are perfected, the space and Earth science communities can perform new and exciting missions unimaginable just a few years ago. In the future, autonomous Space Shuttle and Space Station rendezvous and docking using GPS will become commonplace. Very low cost scientific payloads, such as Spartan, will be deployed from Space Station, fly in

\section{Closed Loop Navigation}

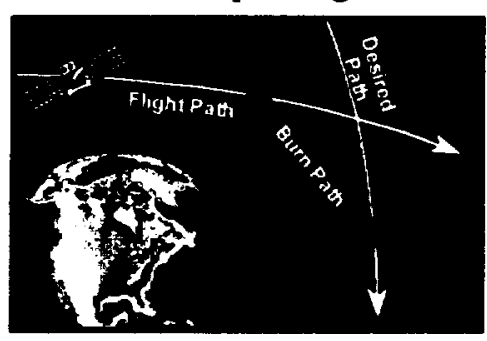

Autonomous Constellation Control

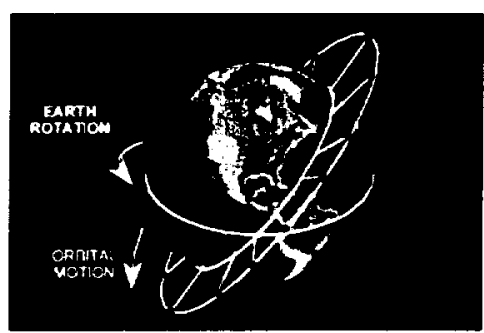

Autonomous Formation Flying of Multiple Satellites

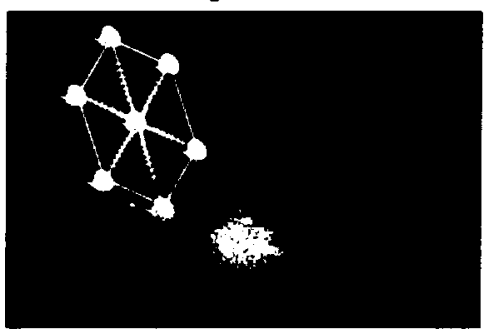

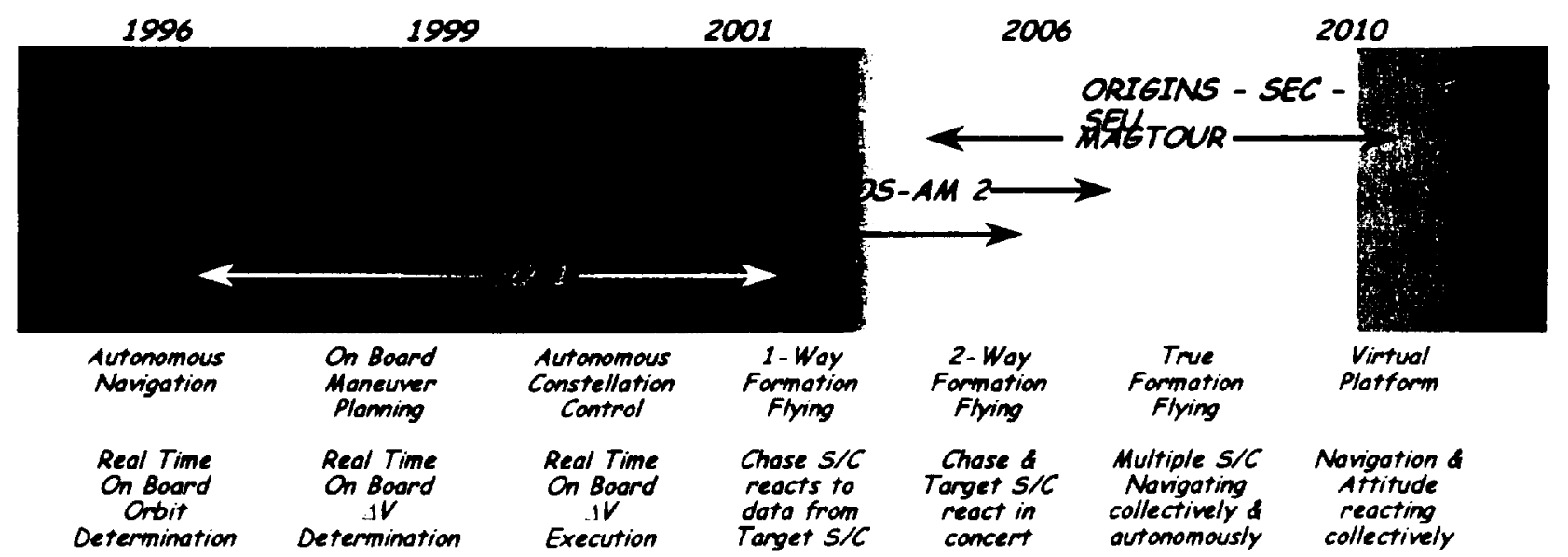

Figure 4: Autonomous Onboard Orbit Determination and Control 
formation, and autonomously return for eventual retrieval. In the process, these enabling technologies will allow synchronous science measurements to occur on multiple space vehicles. Multiple spacecraft formation flying as a virtual platform and gathering concurrent science will soon be feasible once the GPS and formation flying technologies have fully matured.

Ground station automation-The ultimate goal in ground station automation is to develop true "Lights Out" operations. In other words, operating spacecraft with a small team that is only present on the first (daylight) shift. Since the flight and ground segments are intertwined, spaceborne GPS and GPS on the ground are required to enable lower cost "Lights Out" systems. As a result, GPS receivers are being incorporated in ground stations as a timing synchronization source. Ground operations personnel and specific ground passes can be reduced since ranging and Ephemeris uplinks are no longer required. Ground-based formation flying would require a substantial ground personnel requirement. This is not needed if autonomous formation flying is employed.

Further automation of ground stations can occur through the use of systems such as the Transportable Antenna Pointing System (TAPS). In 1994, engineers at GSFC proposed the development of a Transportable Antenna Pointing System (TAPS) using GPS. The TAPS concept integrates a GPS attitude and navigation receiver into a spacecraft antenna pointing system to provide complete autonomy in a ground station operation (see figures 5 and 6). Using GPS, the ground station receives precise time synchronization, 3 dimensional real-time position information (Latitude, Longitude and Height) and attitude sensing relative to an Earth-Centered-EarthFixed coordinate frame-all required to accomplish autonomous antenna pointing to spacecraft. TAPS can also be mounted on a moving vehicle and provide precise antenna pointing despite changes in vehicle direction or vehicle attitude as long as a clear field of view to the satellite is provided. Thus, mobile or portable satellite operations can be accomplished on ships, vans, balloons or airplanes or point small instruments on Space Shuttle Hitchhiker missions. This concept was successfully demonstrated in 1996.

\section{CURRENT AND FUTURE SPACEBORNE GPS RECEIVERS}

The first generation of GPS receivers available to the space community are summarized in Table 1 . These receivers are grouped according to space heritage.
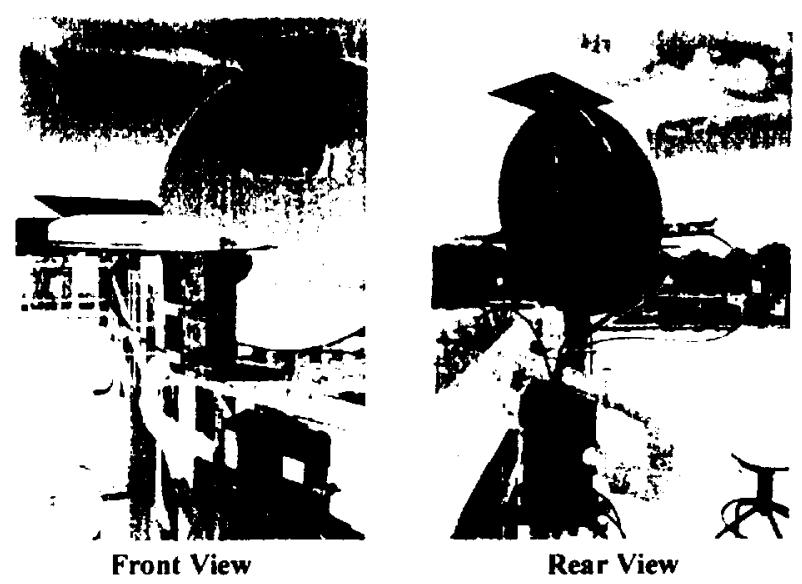

Figure 5: TAPS

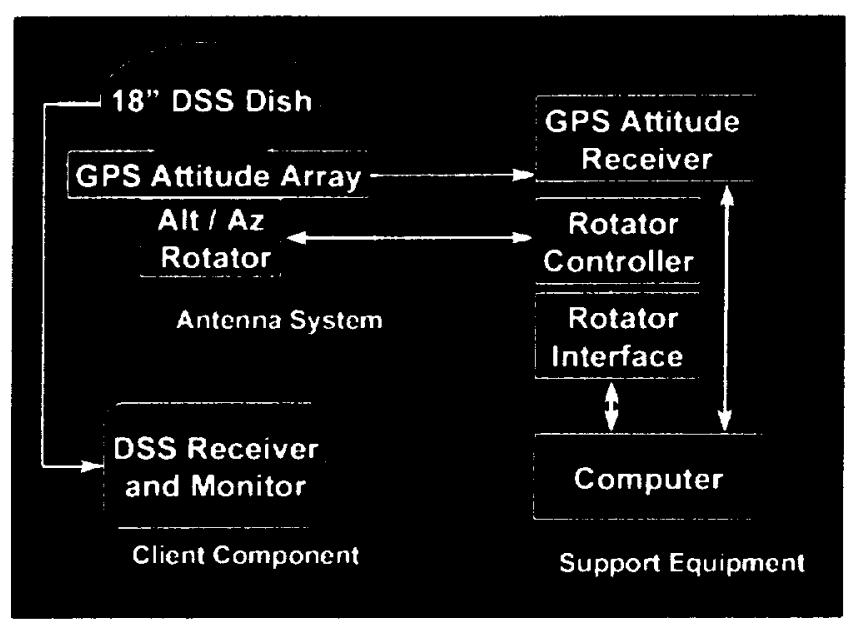

Figure 6: TAPS Block Diagram

Following the figure is a top-level description of the spaceborne receiver's heritage, radiation hardness, and specific capabilities. Spaceborne GPS users should be cautioned that these are all first generation GPS receivers. As such, they are not totally robust. Many are known to possess some algorithmic issues, software bugs, hardware constraints and data outages. These issues are predominantly not GPS manufacturing or design problems. They are more a reflection of the maturity of the technology. They are expected to be corrected in future receiver software releases or when the next set of GPS receivers are available. 
Table 1: Spaceborne GPS Receivers

$\underline{\text { Name }}$

Receivers Flown in Space

Motorola Viceroy

Motorola Monarch

Collins MAGR/S

SS/L Tensor

AOA Turbostar

Trimble TANS Vector

\section{Current Developments}

Honeywell/Trimble/GSFC SIGI

GSFC PiVoT

$\begin{array}{cl}12 & \text { Single } \\ 24 & \text { Single } \\ 12 / 24 & \text { Single } \\ 12 & \text { Dual }\end{array}$

JPL/GSFC/Stanford University

GPS on a Chip
Channels Freg

12

12

5

9

8

6

12
Single

Dual $P(Y)$

Dual $\mathrm{P}(\mathrm{Y})$

Single

Dual $P$

Single
\#Antennas Capabilities

2
2
1
4
1
4

\author{
Navigation, Timing \\ Filtered Navigation, Timing, Rad Hardened \\ Navigation, Timing \\ Attitude, Filtered Nav, Timing, Rad Hardened \\ Precision Nav, Timing, P \& P Codeless \\ Attitude, Navigation, Timing
}

$\begin{array}{cl}4 & \text { Attitude, Navigation, Timing } \\ 2 / 4 & \text { Filtered Nav, Timing, (Attitude Option) } \\ 4 / 5 & \text { Attitude, Filtered Navigation, Timing } \\ 4 & \text { Precision Navigation, Timing, Attitude }\end{array}$

\section{Currently Available Spaceborne Receivers}

\section{Motorola Viceroy}

The Viceroy can now be considered a heritage receiver. It has flown on MSTI-3, Seastar and on a Mir experiment. It is also expected to fly on several future missions, including Earthwatch. As shown in the figure, it is a basic C/A code navigation \& timing receiver. Its electronics were not designed for radiation hardness, but it can withstand a moderate (15 Krad) environment which is adequate for most low Earth Orbiting missions.

\section{Motorola Monarch}

The Monarch is Motorola's premier GPS receiver. It is a 12 channel, radiation hardened $(>100 \mathrm{Krad})$ receiver that is capable of receiving the encrypted $P(Y)$ code. Developed primarily to meet DoD requirements, it can output data in RS-422, MIL-STD 1553 and Remote Interface Unit (RIU) formats. Heritage for this receiver is derived from the $P$ code dual frequency receivers flown on TOPEX and the Extreme Ultraviolet Explorer spacecraft. The receiver has an embedded 1750A microprocessor and the receiver software is written in Ada.

\section{Rockwell/Collins MAGR/S}

The Miniature Airborne GPS Receiver/Shuttle (MAGR/S) is currently the Space Shuttle receiver [1]. This 5 channel receiver is used to provide the Shuttle with real-time navigation and timing. It will eventually replace the use of TACAN during the landing phase of the mission. This is a $P(Y)$ code receiver and NASA Johnson Space Center is using the receiver in its fully secure mode. The receiver is expected to be placed in primary service (the primary Shuttle navigation sensor) in 1999.

\section{SS/L Tensor}

The Space Systems/Loral Tensor [2][3] is a radiation hardened (100 kRad) attitude, orbit and timing receiver. It can accommodate up to four antennas and multiplexes the 9 channel receiver through all four channels to determine vehicle attitude at sample rates up to $10 \mathrm{~Hz}$. A navigation filter is included as a commanded option to improve navigation performance to approximately 100 meters. This receiver has flown on the SSTI-Lewis spacecraft and is expected to fly on the TRW ROCSAT spacecraft, the GSFC EO-1 spacecraft and the SS/L Globalstar constellation.

\section{Allen Osborne Associates Turbostar}

The Turbostar GPS receiver was developed jointly by JPL and Allen Osborne Associates to support NASA scientific investigations in space. This 8 channel, dual frequency $P$ code receiver has a high $(50 \mathrm{~Hz})$ sample rate that enables the device to perform atmospheric and ionospheric sounding measurements. Using cross correlation techniques, the Turbostar can support Pcodeless operation. Similar to the TANS Vector, the Turbostar is a commercial receiver that has been ruggedized for space. The Turbostar has flown on the 
GPS-MET and Wakeshield $2 \& 3$. It is expected to fly on the Danish ORSTED, the South African SUNSAT and the U.S. Geosat Follow-on (GFO) satellites. Using the International GPS Service for Geodynamics (IGS) global network of GPS receivers, the Turbostar receiver can achieve sub-meter post-process orbit determination performance.

\section{Trimble TANS Vector}

The Trimble TANS Vector is a commercial terrestrial receiver that has been ruggedized for use in space. Spaceborne software was developed for this receiver through a partnership with engineers from Trimble, Stanford University, and GSFC. Using this software, the receiver provides 3-axis attitude to approximately 0.5 degree [4][5], navigation to 450 meters and timing to 0.1 microsecond. Approximately a dozen receivers have flown on missions such as Orbcomm, Crista SPAS, REX-II, GADACS, GANE as well as several Space Shuttle Experiments. The receiver is somewhat radiation soft with a tolerance of approximately $8 \mathrm{kRad}$. Despite this, the receiver has supported low Earth orbiting missions for well over a year and in some cases 3 years.

\section{Current Developments for Space}

The current spaceborne GPS receivers available commercially have not kept pace with NASA's current and future requirements. NASA needs receivers that are low cost ( $\$ 100 \mathrm{~K}$ or less), low power and can be reconfigured to meet the specific mission requirements. None of the low cost receivers currently available meet the demanding real-time navigation requirements $(<100$ meters) of many Earth science missions. All the spaceborne receivers have been derived from terrestrial receivers, inheriting the zenith-pointing assumptions that limit the robustness required in spaceborne applications. Therefore, NASA has initiated several in-house GPS development initiatives to bring spaceborne GPS to spacecraft users. These, as well as the low-cost GPS receiver development at Surrey Satellite Technology Limited are described below.

\section{GSFC PiVoT}

An in-house NASA GSFC development is called PiVoT, which stands for Position, Velocity, Time. This modular receiver design is based on the GEC Plessey Chipset [6][7]. PiVoT is expected to be able to withstand a moderate $(15-20 \mathrm{kRad})$ radiation environment. Robust algorithms embedded in PiVoT will allow the receiver to operate in any vehicle orientation and support the demanding navigation requirements of $95 \%$ of NASA's Earth science and space science missions. The design will support up to 4 antennas and an attitude sensing option is planned. The protoflight PiVoT receiver will be delivered in $5 / 98$ with protoflight qualification completed around 10/98.

\section{SIGI Honeywell/Trimble/Collins/GSFC}

The Space Integrated GPS/INS (SIGI) represents a new Inertial Navigation System for NASA's Space Shuttle, International Space Station and Crew Return Vehicle. The INS consists of a Ring Laser Gyro, GPS receiver, inertial navigation computer and power supply mounted in a standard Embedded GPS INS housing.

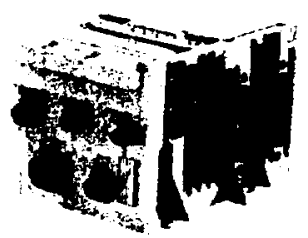

Figure 7: SIGI
See figure 7. Two spaceborne receivers are being produced. The first is the Collins GEM dual frequency receiver. It is both $C / A$ and $P(Y)$ code capable and supports navigation and timing functions. This receiver will be embedded in the SIGI that flies on the Space Shuttle. The second receiver is being developed through a partnership between Honeywell, Trimble and NASA Goddard. This receiver will support the International Space Station and Crew Return Vehicle. The Trimble receiver used in this application supports attitude, navigation and timing. The receiver can track up to 12 satellites. It is an upgrade of the successful TANS Vector receiver. The attitude algorithms and software embedded in this receiver are being developed by NASA GSFC.

\section{JPL/GSFC/Stanford GPS on a Chip}

The GPS-on-a-Chip development effort is a joint, collaborative program with JPL, Stanford University and NASA GSFC as partners. JPL is developing the GPSon-a-Chip hardware and Stanford and Goddard will develop the algorithms, software and validate the system performance through ground-based testing. The development approach was to develop the receiver requirements, design and develop the prototype receiver and make the design available to future industrial partners. The Phase I prototype receiver is expected to be completed by the year 2000 . The GPS on a Chip project started in 1996. GPS-on-a-Chip is being designed to be modular with an open architecture hardware and software design and developed to be flight qualified in the future. GPS-on-a-Chip will support multiple antennas, can be used as a dual frequency $P$ code receiver or a single frequency $\mathrm{C} / \mathrm{A}$ code receiver. It is expected to support attitude, filtered navigation and timing. Prototypes of this receiver are planned to be 
flown on the SAC-C (1999), Champ (1999), and the GRACE (2001) missions.

\section{Surrey Satellite}

Surrey Satellite Technology Limited (SSTL) is also developing a GPS receiver based on the GEC Plessey chipset [6][7]. Similar to the PiVoT design, the SSTL receiver will incorporate radiation protection. The receiver uses an ARM60 32 bit RISC microprocessor. This receiver will support an on-orbit software uplink, a feature that is lacking (but should be required) in many commercial GPS receivers. Data is output via a CAN interface, which is an automobile standard, similar to RS-422. The receiver is being incrementally upgraded and flown as an experiment on several Microsats. The first flight is expected on TMSAT in late 1997 or Early 1998. TMSAT is a Technology Transfer Micro-satellite being constructed for Thailand. This flight will include the receiver in a 2 antenna configuration. The first flight of the $4 / 5$ antenna receiver will be on UoSAT- 12 which is expected to fly in 1998.

\section{7-1999 FLIGHT EXPERIMENT OVERVIEW}

The experiments accomplished prior to 1997 and those planned for 1997-1999 expect to validate some of the initial concepts of using GPS for spacecraft engineering functions and to climb the technological stairsteps depicted in figure 1. Each experiment is unique, providing a wealth of information to the spaceborne GPS receiver design community. These experiments will provide valuable information on using GPS as an autonomous navigation instrument, as a spacecraft attitude determination sensor, as a precise time synchronization a source, and as a relative ranging sensing device.

To date, two spacecraft GPS experiments have flown in 1997. Table 2 outlines the eight Goddard-collaborated spaceborne GPS experiments scheduled for 1997-1999. The table includes the mission name, the spacecraft, the planned experiment objectives, the type of GPS receiver (or receivers) to be flown and the launch date. After reviewing these collaborative experiments, one may observe that there is some overlap in some of the missions. It should be noted that this was done intentionally. The GPS team's intent is to obtain experimental data which is critical to factor into future GPS receiver designs in a timely fashion despite launch vehicle, spacecraft, or experiment failures that are part of the risk of flying in space. The following paragraphs provide more details on each experiment.
GPS Attitude Determination Flyer (GADFLY) Experiment on the SSTI-Lewis Spacecraft

The Global Positioning System (GPS) Attitude Determination Flyer (GADFLY) [8] experiment was launched on the Small Satellite Technology Initiative (SSTI) Lewis spacecraft in August 22, 1997 (see figure 8). The primary objective of the GADFLY experiment was to demonstrate and validate the cost-saving, systems engineering features that can be exploited by using GPS receivers in space vehicles. The experiment's physical hardware included four GPS antennas and pre-amplifiers, cross-strapped to two Space Systems/Loral GPS Tensor receivers. This was expected to be the first long-term, on-orbit flight of a fully space-qualified GPS receiver capable of simultaneously sensing space vehicle attitude, orbit and providing a precise time reference.

The Space Systems/Loral Tensor receivers were flown on GADFLY primarily as an attitude determination experiment. However, an integral part of this experiment was to demonstrate precise time distribution and provide autonomous real-time navigation solutions to the spacecraft subsystems and other experiments. The spacecraft clock used a timing signal and a "time at the tone" message from the GPS Tensor to maintain a timing accuracy to better than two milliseconds. A GPS timing testbed, developed at the Goddard Space Flight Center, was used to test the techniques required to transfer the GPS timing information from the GPS Tensor onto a MIL-STD-1553 spacecraft bus. In addition to the timing information, navigation solutions provided by the GPS Tensor was intended to support the Lewis science objectives. To improve the real-time navigation accuracy delivered by the GPS Standard Positioning Service, the GPS Enhanced Orbit Determination Experiment (GEODE) [9] was being developed for GADFLY. GEODE, an enhanced navigation filter developed at the

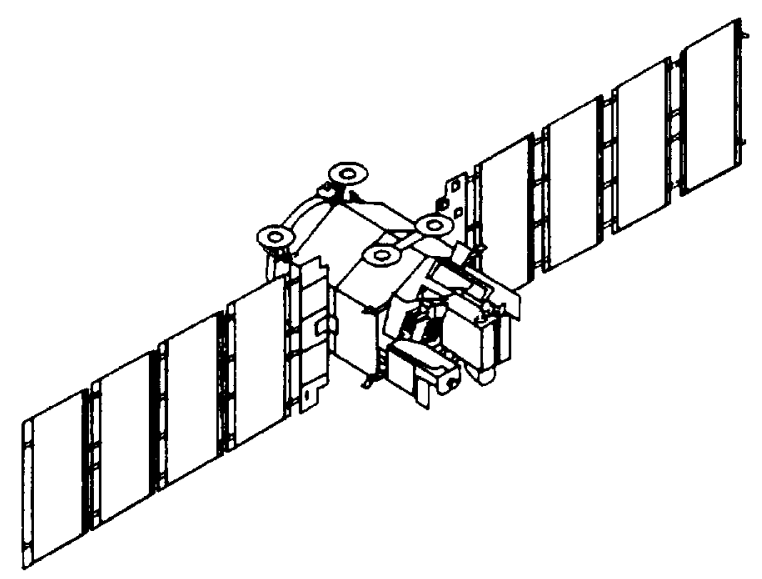

Figure 8: GADFLY on SSTI-Lewis 
Goddard Space Flight Center, is expected to provide navigation accuracy on the order of 10 meters $1 \sigma$. The GADFLY performance goals are shown in table 2 .

The GADFLY team includes members from NASA Goddard Space Flight Center, TRW (the SSTI-Lewis spacecraft manufacturer), Space Systems/Loral, NASA Johnson Space Center, Stanford University, Computer Sciences Corporation, and Orbital.
Table 2: GADFLY Performance Goals

\begin{tabular}{|c|c|c|}
\hline & Spacecraft Requirement & GADFLY Goals \\
\hline Attitude Determination & None (using GPS) & $0.45^{\circ} 3 \sigma$ \\
\hline Orbit Determination & $\begin{array}{l}150 \mathrm{~m} 3 \sigma \text { in-track } \\
150 \mathrm{~m} \text { 3a cross-track } \\
230 \mathrm{~m} 3 \sigma \text { radial } \\
\text { Time Tags: } 2 \mathrm{msec} \text {. } \\
\qquad \mathrm{Hz} \text { update } \\
\end{array}$ & 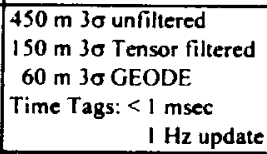 \\
\hline $\begin{array}{l}\text { Precise Timing } \\
\text { Reference }\end{array}$ & 1 msec, $1 \mathrm{~Hz}$ update & \begin{tabular}{|l|} 
Time Tags $<$ I msec \\
Discrete Pulse $<1 \mu \mathrm{sec}$
\end{tabular} \\
\hline
\end{tabular}

Table 3: 1997-1999 Space-Based Experiments

\begin{tabular}{|c|c|c|c|c|}
\hline Experiment & Carrier & Goals & Receiver & Date \\
\hline GADFLY & SSTI-Lewis & $\begin{array}{l}\text { Filtered OD, } \\
\text { Time, RT AD }\end{array}$ & SS/L Tensor & $11 / 5 / 97$ \\
\hline Seastar GPS & Seastar & RT OD, Timing & Motorola Viceroy & $\mathbf{X x} / \mathbf{x x}$ \\
\hline Earthwatch GPS & Earthwatch/Earlybird & $\begin{array}{l}\text { RT AD, Timing, } \\
\text { RT Precise OD }\end{array}$ & $\begin{array}{l}\text { Trimble TANS Vector } \\
\text { Motorola Viceroy }\end{array}$ & $12 / 14 / 97$ \\
\hline SHUCS & STS-91/SPACEHAB & $\begin{array}{l}\text { RT AD, RT OD, Ant. } \\
\text { Pointing, Time }\end{array}$ & Trimble TANS Vector & $5 / 98$ \\
\hline Clark-GPS & SSTI-Clark & RT AD, Filtered OD & Trimble TANS Vector & $6 / 98$ \\
\hline AMSAT GPS & AMSAT Phase IIID & $\begin{array}{l}\text { RT AD, Ops above } \\
\text { GPS Constellation }\end{array}$ & Trimble TANS Vector & $7 / 98$ \\
\hline SAC-A GPS & SAC-A & $\begin{array}{l}\text { RT AD, RT OD, } \\
\text { Spinning AD }\end{array}$ & Trimble TANS Vector & $7 / 98$ \\
\hline $\begin{array}{l}\text { Enhanced Formation } \\
\text { Flying }\end{array}$ & EO-1 & $\begin{array}{l}\text { RT OD }(20 \mathrm{~m}), \text { Time, } \\
\text { AOC, AFF }\end{array}$ & SS/L Tensor & $5 / 99$ \\
\hline GPS on the Shuttle & All Shuttles & RT OD & Collins MAGR/S & Numerous \\
\hline
\end{tabular}

\section{SSTI-Lewis Mission Results}

Prior to the mission, the GADFLY experiment was subjected to a battery of tests to validate that the Tensor receiver was ready for flight. These were successfully completed and the GADFLY team was confident that the experiment would provide and outstanding data return. During the first ground station pass, the Tensor receiver was activated, acquired and locked on to sufficient satellites to produce a navigation and time solution within 20 minutes. The receiver remained active until August 26 when the spacecraft was observed to be spinning at a $2 \mathrm{rpm}$ rate. This unrelated attitude control problem resulted in the total loss of the Lewis mission and a total loss in the GADFLY experiment. Despite this unfortunate setback, the GPS community benefited significantly from the GADFLY experiment. Some of the experiment highlights include: 1) the flight qualification of the Tensor-the first radiation hardened receiver capable of determining attitude, orbit and time; 2) the on-orbit demonstration of the Tensor reciever for a brief period (several days) in a navigation and timing configuration; 3) the development of the GEODE software which is expected to serve over $95 \%$ of all 
Earth orbiting spacecraft; 4) the development of techniques to distribute time through a 1553 bus; and 5) the development of low cost GPS attitude self survey techniques using an antenna fixture. The lessons learned from the Lewis mission are being applied to future flight experiments, including future flights of the Tensor receiver on the TRW ROCSAT and the GSFC EO-I spacecraft.

\section{Seastar}

The Seastar spacecraft, developed by Orbital, includes the Goddard SeawiFS instrument. It was launched on August 1, 1997 and is currently in a $705 \mathrm{~km}$ sunsynchronous orbit. A 12 channel, 2 antenna Motorola Viceroy receiver was flown on this mission to support mission orbit determination and vehicle timing. The use of GPS on this mission was not actually a flight experiment but a mission-critical capability. Prior to the flight, Motorola and Orbital recognized the need to fully understand and characterize how the receiver would perform in space. They requested the use of the GSFC GPS facility[10] to independently validate the Viceroy receiver performance. The GSFC GPS facility employs a 40 channel Nortel GPS simulator. The Viceroy was put through a battery of tests, which proved to be critical in improving the receiver's performance. As a result of this testing, software modifications were made prior to flight which have permitted the Seastar spacecraft to provide outstanding Earth science measurements. Thus, preflight testing of GPS in a simulator environment is crucial when the receiver is to be used as a critical navigation device.

\section{EarthWatch-Earlybird}

The Earlybird-1 satellite is being built by Orbital/CTA for EarthWatch, Inc. This spacecraft represents EarthWatch's first entry into the commercial Earth imaging satellite business. This mission will be placed in a $470 \mathrm{~km}$, sun synchronous orbit. Of particular interest to the GPS community is that this spacecraft will flying two different GPS receivers-the Motorola Viceroy and the Trimble TANS Vector C/A code receivers. The TANS Vector, using four antennas, will provide attitude, orbit and time data to the vehicle and the Viceroy will provide orbit and time information to the spacecraft. This mission has a very aggressive $3-5 \mathrm{~m}$ (1-sigma) orbit accuracy requirement. A team consisting of members from Orbital, University of Colorado, and Van Martin Systems [11] have developed a low cost, high accuracy Orbit Determination System for this mission. This system employs a MicroCosm precision orbit software system, derived from NASA Goddard's GEODYN II, and the use of the International GPS
Service for Geodynamics (IGS) [12] ground system. Using this system, Davis, et. al. achieved 3-7 m orbit accuracies using single frequency carrier phase data from a Trimble receiver in the $800 \mathrm{~km}$ RADCAL [13] orbit. The GPS team also hopes to get high accuracy attitude data from this mission since the spacecraft includes high accuracy attitude sensors on-board that can be used to calibrate the GPS attitude data. The Earlybird-1 spacecraft is tentatively scheduled for launch on December 14, 1997. The GPS collaborative experiments on this mission are sponsored by EarthWatch, Orbital/CTA, GSFC, Trimble, Motorola, University of Colorado, Van Martin Systems and Microcosm.

\section{Clark-GPS}

In addition to the GADFLY experiment on the SSTILewis spacecraft, a GPS experiment will also be included on the SSTI-Clark spacecraft [14], which is currently slated to be launched in June, 1998 (see figure 9). The objectives of the Clark-GPS experiment is to study real-time GPS orbit and attitude determination. The experiment's physical hardware includes four GPS antennas and pre-amplifiers, cross-strapped to two Trimble TANS Vector GPS receivers.

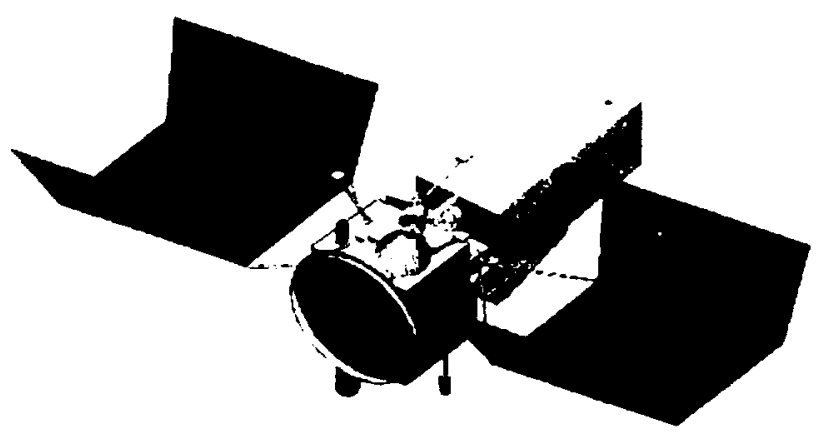

Figure 9: SSTI-Clark

There are six cases of interest that will be studied with the Clark-GPS orbit determination experiment. The GPS Operational case will study the use of an onboard estimator and periodic updates to improve the orbit accuracy from the base GPS Standard Positioning Service (SPS) accuracy of 150 meters, $1 \sigma$, to better than 25 meters, $1 \sigma$. The GPS Dropouts case will look at the GPS data dropouts that occur in various portions of the spacecraft orbit, particularly at high altitudes where visibility of GPS satellites is at its worst. Degradation of the orbit estimator propagated orbit position during these dropouts will also be characterized. The third case that will be studied is the ability of the orbit estimator to reject GPS Outliers through the use of a chi-square test. 
The fourth case to be studied for the Clark-GPS orbit determination experiment is the performance of the GPS receiver and the orbit propagator during and after orbitadjust maneuvers. The case of an inoperative GPS receiver is also considered, with the ability on the spacecraft to switch to the redundant GPS receiver and reinitialize the orbit estimator by ground command. Finally, the case of autonomous initialization of the orbit estimator after safehold will also be studied.

The Clark-GPS attitude determination experiment will study the generation of GPS attitude for a spacecraft in earth-pointing mode. As a long-term attitude determination experiment, the static and dynamic error sources of GPS attitude can be characterized by comparing them to more accurate attitude information from SSTI-Clark's star tracker and other spacecraft sensors. The impact of vehicle multipath on GPS attitude determination accuracy can also be investigated.

The Clark-GPS experiment on SSTI-Clark has been put together by a team consisting of CTA, Welch Engineering, and NASA Goddard Space Flight Center.

\section{AMSAT-GPS}

AMSAT Phase 3D is the latest in a long series of satellites built by the Radio Amateur Satellite Corporation (AMSAT). It is typical of AMSAT satellites in that it is being built almost entirely by a world-wide volunteer staff of amateur radio operators and satellite enthusiasts from AMSAT, the NASA Goddard Space Flight Center, and others throughout the world. The Phase 3D satellite is scheduled for launch as a secondary payload on an Ariane 5 flight in 1998.

The AMSAT-GPS experiment will be the first to study the use of GPS signals above the GPS constellation. AMSAT Phase 3D will be in a 4,000 $\times 47,000$ kilometer Molniya orbit, its apogee well over the 20,000 kilometer altitude of the GPS satellites. In addition to providing further long-term, real-time GPS attitude and orbit determination experience, the AMSAT-GPS experiment will be able to map the GPS constellation signal patterns available above the constellation, as well as give an understanding of the robustness and limitations of making use of GPS in this region.

The hardware for the AMSAT-GPS experiment will consist of two Trimble TANS Vector GPS receivers, along with two sets of four GPS antennas. Four patch antennas will be located on the perigee side of the spacecraft, while four high-gain antennas will be placed on the apogee side.

\section{SPACEHAB Universal Communications System (SHUCS)}

The SPACEHAB Universal Communications System (SHUCS) uses a flat panel L-band phased array antenna mounted to a two-axis pointing system to communicate through an Inmarsat satellite. SHUCS is mounted on top of SPACEHAB. SPACEHAB is a reusable commercial habitation module that is flown in the Orbiter cargo bay. This system, similar to the TAPS system described previously, allows customers to receive $64 \mathrm{kbps}$ of data directly and send data commands in real-time; bypassing the NASA communications network. The antenna pointing system is controlled by a Trimble TANS Vector navigation and attitude receiver provided by NASA Goddard. The SHUCS experiment is planned to fly on STS-91 in May 1998. This represents the first time a spaceborne pointing system will use GPS to steer an antenna to a target. During the flight, GPS receiver and SHUCS operational data will be provided to the SPACEHAB and Goddard experimenters in real-time through the Inmarsat/SHUCS link-up. An educational outreach program has been built into this experiment to allow the Goddard obtained flight data to be available to students in real time through a World Wide Web site.

\section{SAC-A}

The SAC-A spacecraft is a small free-flying satellite designed by CONAE in Argentina. SAC-A will be deployed from a hitchhiker bridge mounted in the Space Shuttle Endeavour in July 1998. The SACA GPS experiment is expected to perform the first ever attitude and navigation sensing on a spinning

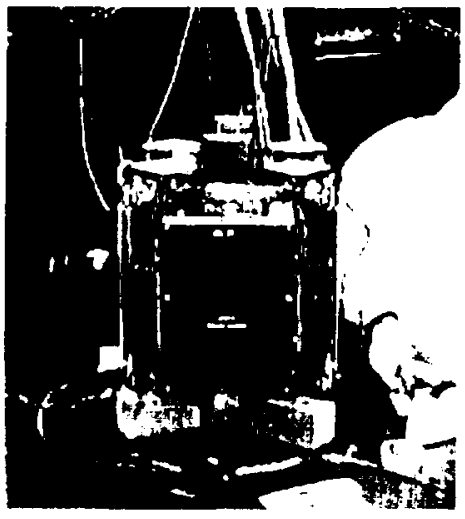

Figure 10: SAC-A spacecraft. As shown in figure 10, four patch antennas with a very short baseline $(<0.5$ meter) are mounted on the top of the SAC-A spacecraft. A Trimble TANS Vector receiver will be employed on SAC-A to determine the vehicle attitude, orbit and time. This is a collaborative partnership between CONAE in Argentina and the NASA Goddard Space Flight Center. 


\section{EO-1 Enhanced Formation Flying Experiment}

The primary objective of the enhanced formation flying experiment on the New Millenium Program (NMP) Earth Orbiter-1 (EO-1) mission is to demonstrate onboard autonomous relative navigation and formation flying control between the EO-1 and Landsat-7 spacecraft. An automated mission design and automated maneuver planning tool, AUTOCON, which was developed by $\mathrm{AI}$ Solutions under direction by the Goddard GN\&C team, has been used for operational mission design. AUTOCON is being modified to operate onboard the spacecraft to support autonomous constellation control and formation flying. This will be accomplished by having the flight control system plan a maneuver that places EO- 1 within 1 minute of separation from Landsat7 and then maintain that separation to a tight tolerance of 6 seconds for an extended period of time.

The algorithms and software tools for this demonstration will be developed using a modular approach so that they can easily be used onboard future Earth orbiting missions. These algorithms [15] will be implemented using fuzzy logic engines for constraint checking and control of the formation flying algorithms.

The key benefits of this enhanced formation flying technology are to eliminate routine ground maneuver planning and commanding requirements, reduce costs, enhanced science capabilities, and to advance the technology for complete lights-out application for the New Millennium Program. The system will provide a real-time low-cost formation flying control with the flexibility to meet a broad range of mission requirements including ground track, inclination, and altitude control as individual or multiple spacecraft requirements.

\section{Space Shuttle Flights}

Since 1993, engineers at the NASA Johnson Space Center have sponsored several different experiments to validate the concepts required to fly GPS as an in-line avionics component on the US Space Shuttles. The current phase of Shuttle/GPS operations is the use of a Precise Positioning Service (PPS) receiver as a single string navigation device. A Rockwell Collins MAGR/S [1][16] receiver was chosen for this phase of the development effort. During the flights, the navigation data is downlinked to the ground and is available in realtime on the astronaut crew displays. During landing, the GPS system performance will be compared to the results obtained by TACAN, the primary Shuttle navigation device. The single string GPS system is currently incorporated on 3 Shuttles (Discovery, Atlantis and
Endeavour). It was first demonstrated on the STS-79 mission which was launched on September 16, 1996.

The final phase of the Shuttle GPS validation is to incorporate a three string GPS on all four Orbiters. Since the Shuttle avionics systems requirement is to be two fault tolerant, a minimum of three GPS receivers are required per Space Shuttle. Once this final phase has been completed and the system validated, TACAN service will be removed for future Shuttle Missions and the GPS antennas will be installed in place of the TACAN antennas. Currently, TACAN navigation is provided for the Shuttle within 300 miles of the landing site. The first flight of the GPS-only navigation solution will be on STS-92, which is tentatively scheduled for January 1999.

Using GPS for Space Shuttle Navigation will allow the Shuttle to eliminate its need for TACAN navigation. In addition, GPS is expected to improve on-orbit navigation performance and save approximately \$3-4 Million per year by eliminating the need for Microwave Landing System ground stations.

\section{CONCLUSIONS}

Significant systems cost, power, and weight savings, as well as enhanced vehicle autonomy are expected from exploiting GPS technologies in future space vehicles. Several spaceborne GPS receivers have been developed and several others are being developed to ensure that future spacecraft and constellations of spacecraft can operate with minimal ground intervention. A combined industry, university and government team of partners have defined the vision and direction for spacebome GPS and are implementing this vision in the most cost effective manner.

\section{REFERENCES}

[1] Murray, S.V., "Global Positioning System for the Space Shuttle", Presented at NASA GSFC, February, 1996.

[2] Fuller, R.A., Kemper, B., and Rodden, J.J., "Spacecraft Guidance and Control with GPS TENSOR", $19^{\text {th }}$ Annual AAS Guidance and Control Conference, Breckenridge, CO, February 7-11, 1996.

[3] Sacchetti, A., "GPS for Orbit and Attitude Determination: Hardware Design and Qualification Plan for a Spaceborne Receiver," ION-GPS-94, Salt Lake City, UT, September 20-23, 1994. 
[4] Freesland, D., et. al., "GPS Based Attitude Determination, The REX-II Experience", AIAAVUSU Small Satellite Conference, Logan, UT, September 1996.

[5] Lightsey, E.G., et. al., "Flight Results of GPS Based Attitude Control on the REX-II Spacecraft ", ION-GPS-

96, Kansas City, MO.

[6] Theroux, Y., "Open Architecture Design for GPS Applications," ION-GPS-95, Paim Springs, CA, September 12-15, 1995.

[7] Clark, T., Varney, D. and Bauer, F., "A Low Cost Spacecraft Receiver for Orbit and Attitude Determination," ION-GPS-94, Salt Lake City, UT, September 20-23, 1994.

[8] Bauer, F. H., et. al., "The GPS Attitude Determination Flyer (GADFLY): A Space-Qualified GPS Attitude Receiver on the SSTI-Lewis Spacecraft", ION-GPS-95, Palm Springs, CA.

[9] Hart, R.C., et. al. "Global Positioning System (GPS) Enhanced Orbit Determination Experiment (GEODE) on the Small Satellite Technology Initiative (SSTI) Lewis Spacecraft", ION-GPS-96, Kansas City, MO.

[10] O’Donnell, J. R., Jr., et. al., "Testing of GPS-Based Attitude Control Systems”, ION-GPS-96, Kansas City, MO.
[11] Davis, G.W., et. al., "A Low Cost, High Accuracy Automated GPS-Based Orbit Determination System for Low Earth Satellites", ION-GPS-97, Kansas City, MO.

[12] Beutler, G., and Neilan, R., International GPS Service for Geodynamics Resource Information, January 1996.

[13] Lightsey, E.G., et al., "Application of GPS attitude Determination to a Gravity Gradient Stabilized Spacecraft," ION-GPS-93, Salt Lake City, UT, September 1993.

[14] Freesland, D., et. al., "SSTI-Clark Attitude Determination and Control Subsystem Status Review", CTA Design Review, January 1996.

[15] Bauer, F.H., Bristow, J., Folta, D., Hartman, K., How, J. and Quinn, D., "Satellite Formation Flying using an Innovative Autonomous control System (Autocon) Environment", AIAA GN\&C Conference, New Orleans, LA, August 1997.

[16] Kirpes, R.R., and Burkle, M.W., "Ballistic Propagation of the MAGR-Shuttle Navigation Solution", ION Proceedings of the $52^{\text {nd }}$ Annual Mtg, Cambridge, MA, June 1996. 